\title{
Virtual Torque Sensing for Multirotor Propulsion Systems
}

\author{
Bart Theys $^{1}$ and Joris De Schutter ${ }^{2}$
}

\begin{abstract}
This paper presents a method to sense propeller torque of multirotors without additional torque sensors by using the measured input voltage, throttle setting and rotational speed. Torque, rotational speed and current of a brushless direct current motor with electronic speed controller are measured on a setup using seven different propeller diameters, seven input voltages and 20 throttle settings. The resulting rotational speed, torque and current are measured to create a data set spanning the feasible operating range of this motor with speed controller. A model based on only four parameters is proposed and trained on the data of only one propeller and the data without propeller. This model results in torque errors less than $0.01 \mathrm{Nm}$ for $90 \%$ of the data set. For the highest accuracy, the whole data set is re-mapped into a practical 3D lookup table using local fits and results in torque errors less than $0.005 \mathrm{Nm}$ for $90 \%$ of the data set.
\end{abstract}

\section{INTRODUCTION}

Multirotors or 'drones' are popular aerial platforms because of their mechanical simplicity. More and more they are used for high-demanding missions exploiting their full potential such as urgent medical deliveries, offshore inspections, human transport and even racing. An accurate model of the multirotor and its sub-systems is required for simulation, model based controller development, mission optimization, system monitoring and design or customization of the system.

Mechanically, a multirotor can be split into a fixed structure or 'body' and the rotating propellers [1]. Ignoring the aerodynamic effects of the propeller slipstreams, interaction between body and propellers consists of three forces and moments and rotational speed of the motor shaft to the propeller hub as shown in Fig. 1.

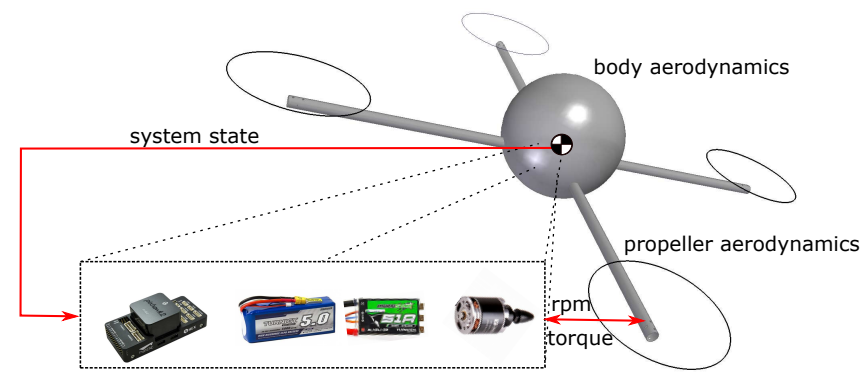

Fig. 1. Overview of multirotor consisting of a body, propellers and motors with ESC connected to the flight controller and battery.

\footnotetext{
${ }^{1}$ Bart Theys is with the Department of Mechanical Engineering, Robotics Research Group, KU Leuven \& Core Lab ROB Flanders Make, 3000 Leuven, Belgium bart.theys akuleuven. be

${ }^{2}$ Joris De Schutter is with the Department of Mechanical Engineering, Robotics Research Group, KU Leuven \& Core Lab ROB Flanders Make, 3000 Leuven, Belgium
}

During hover or low speeds, the main force and moment acting on the motor shaft are the propeller thrust and torque which can be measured relatively easy on a static test bench. The rotational speed is easily monitored through the commutation of the three phases of the motor. However, the three forces and moments acting on the motor shaft during forward flight cannot be measured directly without additional sensors. These forces and moments were modelled by studies as [2], [3] and [4] relying on data from wind tunnel data sets for axial flow only, as from [5], or from sparsely available data presented in [6], [3] and [7] for other flight phases. Wind tunnel tests however require specialized infrastructure and are not completely representative for free flight conditions of a multirotor because of wall effects and the need for a physical mount. In order to create an accurate model of the multirotor propeller, ideally, all forces and moments on the propeller would be measured in flight. Davis and Pounds [8] mounted a sensor between each motor and the frame of a multirotor that allowed them to calculate the vertical and horizontal component of the thrust.

The principle behind in-flight propeller modelling and identification was already demonstrated by Sartori [1] who identified thrust, drag and side force on propellers from mostly planar indoor flights. Earlier also Gill [9] performed propeller identification based on a tethered experiment with a multirotor allowing it to reach higher speeds indoor. Six [10] identified the propeller coefficients from flight data of a hovering quadrotor. Steps towards modelling of the complete multirotor at higher speeds in forward flight were presented in [11] and [12] while [13] presented the modeling of propeller interactions. To facilitate the in-flight identification of a multirotor and its sub-systems, from the three forces and moments that the propeller produces, the torque produced by the brushless direct current (BLDC) motor can be calculated based on available sensor data logged on the flight controller during flight. In robotics, torque of an electromotor is often estimated based only on its current [14]. For example [15] uses a third-order polynomial model to experimentally identify the relation between torque and current of BLDC motors of a KUKA KR150.

For multirotor propulsion systems, the BLDC motors are controlled by an electronic speed controller (ESC), that modulates the voltage from the battery to the three phases of the motor and controls speed by decreasing the pulse widths for varying throttle signals. An identification of this motor with ESC was done by Gong in [16] and [17], showing efficiency maps of motor and ESC separately at different input voltages using a 3-phase power analyser. They reported a decrease in efficiency for higher currents 
and lower throttle settings. With a three-parameter motor model and a four-parameter ESC model they accurately model the efficiency of the tested motors and ESCs but without distinguishing between voltage or current losses that influence the rotational speed and torque of the motor. Other BLDC motor models like described in [18] use four constants to take viscous damping losses and Eddy currents into account. [19] used a polynomial fit to model the losses or [17] and [20] used three constants from which the latter considered these to be a linear function of the input voltage.

Complementary to the aforementioned studies, this paper presents a model based method to calculate propeller torque from the logged on-board flight data containing rotation speeds, battery voltages and throttle settings throughout the flight. Besides the use for identification of the propeller and the whole multirotor system, torque estimation can also be used for torque control, system monitoring and benchmarking. To calculate the torque, two approaches are applied to a data set obtained as described in section II. Section III presents a novel model for the ESC in combination with a DC motor model for the BLDC motor that fits the whole feasible operating range and requires only a limited amount of test data. Section IV presents an interpolation approach resulting in the highest accuracy when a large set of test data is available.

\section{EXPERIMENT}

This section describes how the data set spanning the feasible operating range for a motor with ESC was created and presents the test results that will be used later for identification.

\section{A. System overview and test setup}

Fig. 2 and table I present the overview of the test setup. A power supply provides a stable constant voltage to the sensor setup [21] which measures the voltage $U_{b a t}$ and current $I_{b a t}$ at the battery side of the ESC. A pair of load cells allows to measure the torque produced by seven different propellers $Q_{\text {load }}$ that can be mounted on the shaft. Flexible motor wires are guided along the extension of the motor shaft to minimise any possible torque transfer. Although thrust of the propeller is also measured with a load cell, it is not used or required for this paper. The control board executes a test sequence that applies the desired throttle settings to the ESC. An optical sensor measures the rotational speed $\omega$ of the motor with a piece of reflective tape.
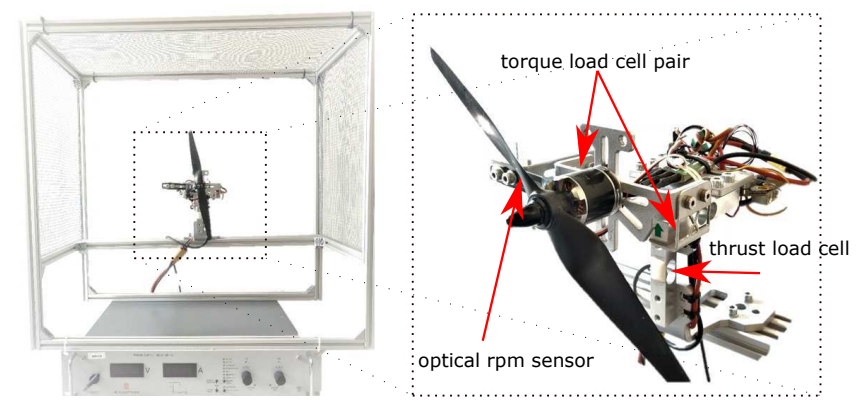

Fig. 2. Overview of the test setup.

TABLE I

OVERVIEW OF COMPONENTS USED

\begin{tabular}{|c|c|}
\hline power supply & Delta Elektronika SM52-AR-60 \\
\hline ESC & Turnigy Multistar opto 51A 32bit \\
\hline motor & T-motor LT2216-11 KV900 V2.0 \\
\hline sensor setup & $\begin{array}{c}\text { RC-Benchmark 1580 with rigid } \\
\text { hinges and RC control board [21] }\end{array}$ \\
\hline propellers & GWS 5x3 \\
& GWS 'bullnose' 5x4.5 \\
& custom cut 6 inch propeller \\
& Graupner e-prop 8x5 \\
& Graupner e-prop 9x5 \\
& Graupner e-prop 10x5 \\
& Graupner e-prop 11x5 \\
\hline
\end{tabular}

\section{B. Test procedure and data processing}

To practically apply a torque to the motor, seven consecutive sizes of propellers are mounted after starting without a propeller. After mounting the propeller, an input voltage is gradually set from $12 \mathrm{~V}$ to $24 \mathrm{~V}$ in steps of $2 \mathrm{~V}$. Finally, throttle is set in 20 steps from zero to full throttle and back with the average of 20 samples recorded after 2 seconds of settling time for each throttle step. A measurement at zero rotational speed right before and after each throttle cycle is used to mitigate any offset or possible sensor drift during each test cycle while taking the average from the measurements with the same throttle setting eliminates possible hysteresis.

To get an idea of the accuracy of the torque measurement and the repeatability, Fig. 3 shows the measured torque as a function of the measured rotational speed $\omega$ for three identical propellers in different test cycles. A quadratic fit on the top graph allows to visualise the relative variation of the measured data to this averaged fit on the bottom graph. Next to some hysteresis, offsets between the experiments of up to $5 \%$ are observed for torque as well as current.

\section{Test results}

Fig. 4 shows all the points of the data set with torque and current presented as a function of the throttle setting, voltage and rotational speed. On these graphs, each combination of voltage and throttle setting has eight decreasing rotational speeds resulting from the increasing loads except for the two largest propellers for which the maximum applied throttle 

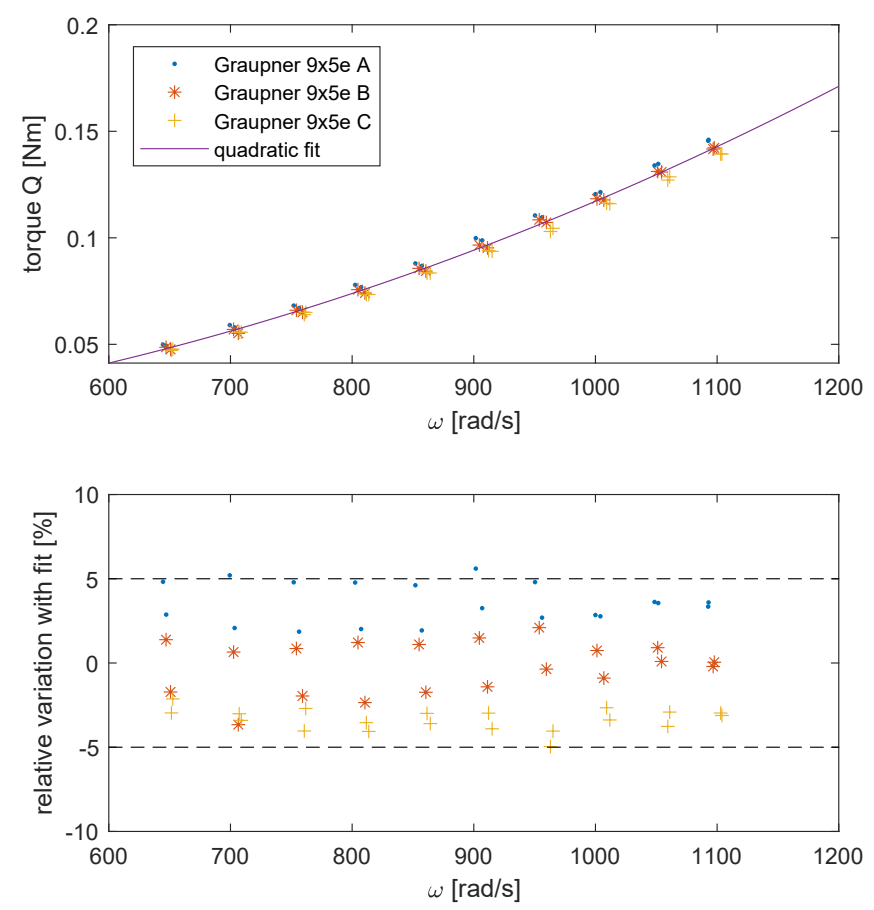

Fig. 3. Measured torque as a function of $\omega$ for three identical propellers shows relative variations between experiments up to $5 \%$.

setting was limited to $80 \%$ to prevent overheating of the motor.
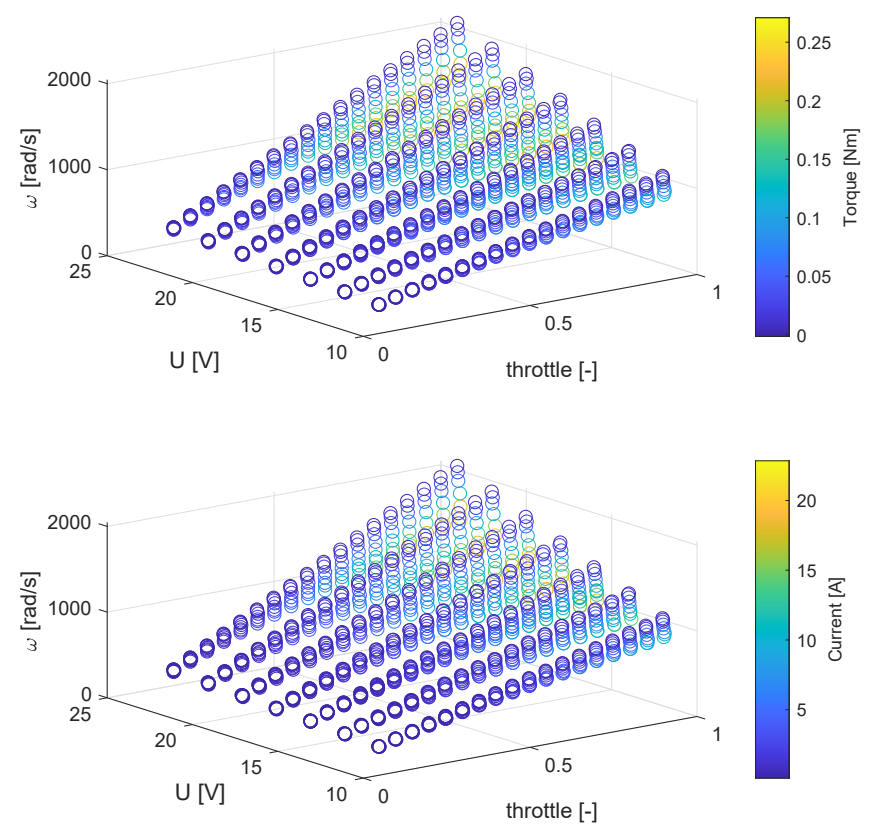

Fig. 4. The whole data set presented with torque and current in function of the battery voltage, throttle setting and rotational speed.

The decreasing rotational speeds for higher loads at constant voltage and throttle setting are presented in more detail by Fig. 5. This graph shows that this ESC, like most others used for multirotors [22], does not control the rotational speed in a closed loop but reduces the average voltage to the motor $U_{m o t}$ by rapidly switching on and off the battery voltage. The proportion of "on" time per switching cycle is referred to as "duty cycle" $D$ resulting in:

$$
U_{m o t}=D U_{b a t} \quad[V]
$$

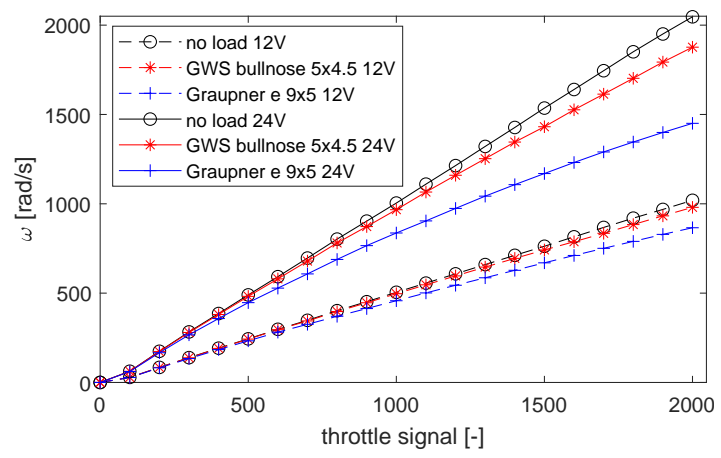

Fig. 5. Decreasing $\omega$ for higher loads shows that ESC does not control $\omega$ in a closed loop.

The relation between the duty cycle and the value of the throttle setting $t$ depends on the used control scheme in the firmware of the ESC. For this ESC a perfectly linear relation between rotational speed and throttle setting is observed for the no-load data, therefore the following model linear relationship is used:

$$
D=\frac{t-t_{\min }}{t_{\max }-t_{\min }}[-]
$$

with $t_{\min }=40$ and $t_{\max }=2047$. It must be noted that many ESCs are controlled using a $50 \mathrm{~Hz}$ pulse width modulated signal with throttle signals typically ranging from $1000 \mu s$ to $2000 \mu s$. This particular ESC is controlled with a "DShot150" communication protocol because this allows to directly communicate the rotation speed back to the flight controller.

An ideal ESC realises this decrease in voltage from battery to motor without any energy losses. Hence the current through the motor $I_{m o t}$ would increase accordingly:

$$
I_{m o t}=\frac{I_{b a t}}{D} \quad[A]
$$

However, from the data set we observe that losses occur due to the ESC regulating $U_{m o t}$ with more losses for lower duty cycles required at higher input voltages $U_{b a t}$, presented by Fig. 6. The efficiency of the motor with ESC $\eta_{m o t+e s c}$ calculated as

$$
\eta_{\text {mot }+e s c}=\frac{Q_{\text {load }} \omega}{U_{\text {bat }} I_{b a t}}[-]
$$

is plotted as a function of the propeller speed $\omega$ for a range of different input voltages. Fig. 6 shows a decrease in efficiency when a higher input voltage is applied for the same desired $\omega$ of the propeller, hence same mechanical power. For example, driving the propeller at $800 \mathrm{rad} / \mathrm{s}$, the efficiency drops from $82 \%$ to $76 \%$ when doubling the voltage from $12 \mathrm{~V}$ to $24 \mathrm{~V}$. Therefore, losses due to the ESC limiting the power cannot be ignored. 


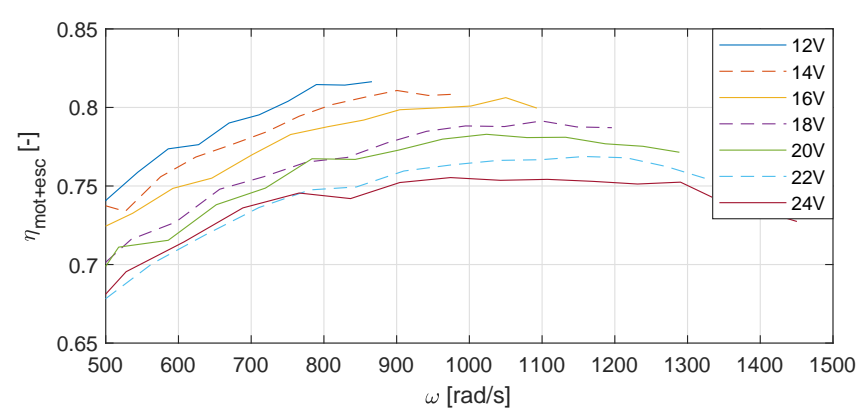

Fig. 6. Decreased efficiency when using a higher input voltage to drive a Graupner 9x5e propeller.

\section{GLOBAL IDENTIFICATION}

This section describes a model for a BLDC motor with ESC which is used to fit the whole range of the data set. From this model, torque and current are calculated based on the input voltage, throttle signal and rotational velocity. A minimal set of parameters is identified and the model is validated on its accuracy in predicting torque and current without sensors.

\section{A. Model}

As presented in the introduction, many models for DC motors and BLDC motors with ESC were proposed in literature. The motor is most commonly modelled using a three-parameter DC electric motor model [24] described by following equations:

$$
\begin{gathered}
Q_{\text {mot }}=Q_{\text {load }}+Q_{f}=K I_{\text {mot }}[N m] \\
e=K \omega[V] \\
U_{m o t}=e+I_{\text {mot }} R[V]
\end{gathered}
$$

with $Q_{m o t}$ the torque produced by the motor and $e$ the backEMF of the coils. In this model, the motor constant is related to the more commonly used " $K V$ " as specified by the motor manufacturer:

$$
K=\frac{30}{\pi K V} \quad[V s / r a d]
$$

$R[\Omega]$ represents the internal resistance of the motor windings. Any remaining losses such as friction are captured by $Q_{f}$. These remaining losses are also often represented by a no-load current [17]:

$$
I_{0}=\frac{Q_{f}}{K} \quad[A]
$$

This DC motor model in combination with equations (1-3) is not able to capture the losses due to the ESC controlling the motor voltage as was shown in Fig. 6. Additional losses can occur such as losses in the electronics due to the constant switching of the transistors [20], the modulated voltage to the motor causing the motor to run with lower efficiency than when it is fed with a smooth continuous voltage [20], magnetic hysteresis [18] and power required by the ESC control circuitry. After comparing the possible nature of the current and voltage losses to the trends in this visualised data, two additional constants are defined. The parameter representing the internal resistance $R$ in (7) is modelled as linearly increasing with the input voltage by a factor $a$.

$$
R=R_{0}+a U_{b a t}[\Omega]
$$

Additionally current losses that linearly increase with the input voltage by a factor $b$ are introduced, replacing (3):

$$
I_{m o t}=\frac{1}{D}\left(I_{b a t}-b U_{b a t}\right) \quad[A]
$$

This linear increase of the internal resistance of the motor model and an ESC current loss linear with the input voltage succeeded in capturing all losses, without distinguishing between the precise origin of these losses.

\section{B. Validation}

The model of the ESC with motor contains four parameters $K, R_{0}, a$ and $b$ to be identified such that the model fits the data as close as possible. In this paper, the objective function is chosen

$$
o b j=\sum \frac{\left(I_{m}-I_{f}\right)^{2}}{\overline{I_{m}}}+\frac{\left(Q_{m}-Q_{f}\right)^{2}}{\overline{Q_{m}}}
$$

with subscript $m$ for the measured data points and $f$ for the fitted points.

First the four parameters of the model are identified using all data except for the Graupner e 9x5 propeller. Fig. 7 shows the distribution of the absolute model error on current and torque for the whole data set. The identified parameters are $K V=840.5 \mathrm{rpm} / \mathrm{V}, R_{0}=0.1484 \Omega, a=0.0056 \Omega / V$ and $b=0.0134 \mathrm{~A} / \mathrm{V}$.

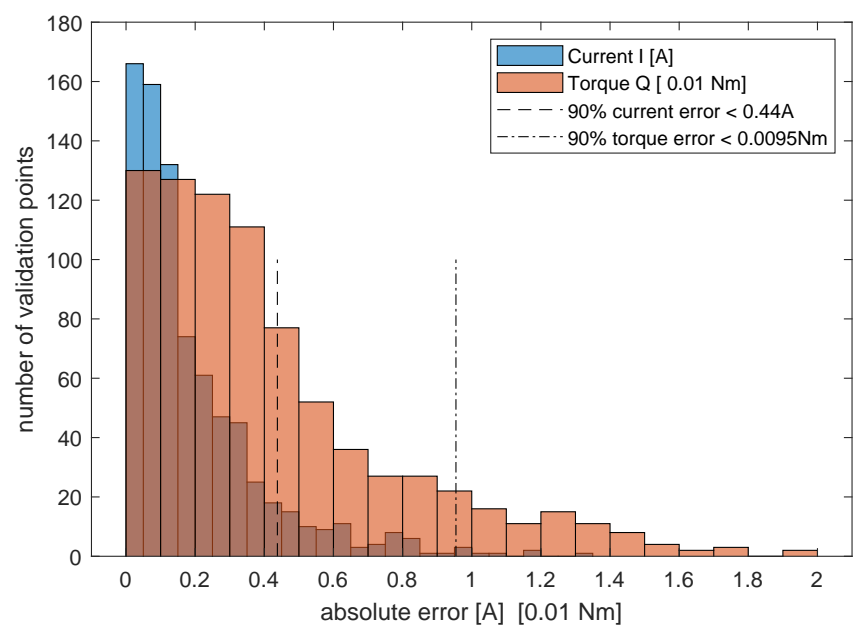

Fig. 7. Absolute current and torque error of the four-parameter model trained and validated on the whole data set.

The model fits well to the whole data set. Fig. 8 shows the errors of this model for the Graupner e 9x5 propeller only, that was not used for the identification. The errors are smaller than for the whole data set which can be explained by the fact that the largest absolute errors occur at the highest loads from the largest tested Graupner $11 \times 5$ propeller. 


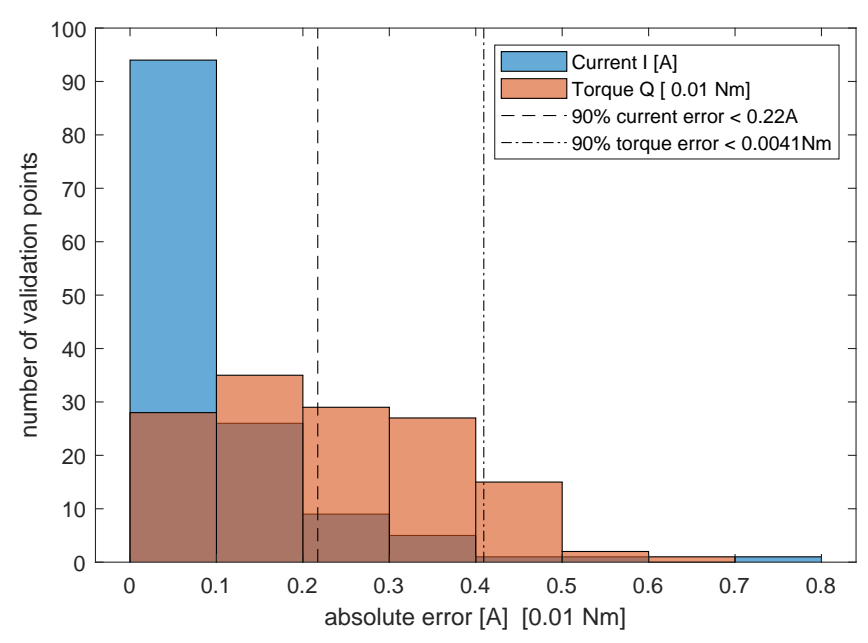

Fig. 8. Absolute current and torque error of the four-parameter model with only the Graupner e 9x5 propeller data as validation.

The purpose of the general model is to simulate the motor with ESC for its whole operating range without testing this whole range on a test bench. Therefore, the data from only one propeller and the no-load case are used to determine the model parameters: $K V=840.5 \mathrm{rpm} / V, R_{0}=0.1565 \Omega$, $a=0.0054 \Omega / V$ and $b=0.0187 A / V$. These parameters closely match the previous set. Fig. 9 shows the absolute current and torque error from this model fit on the whole data set. For $90 \%$ of the data set, the absolute error on estimated current is less than $0.5 \mathrm{~A}$ and torque less than $0.0106 \mathrm{Nm}$ which is only slightly larger compared to a fit on the whole data set as was shown in Fig. 7

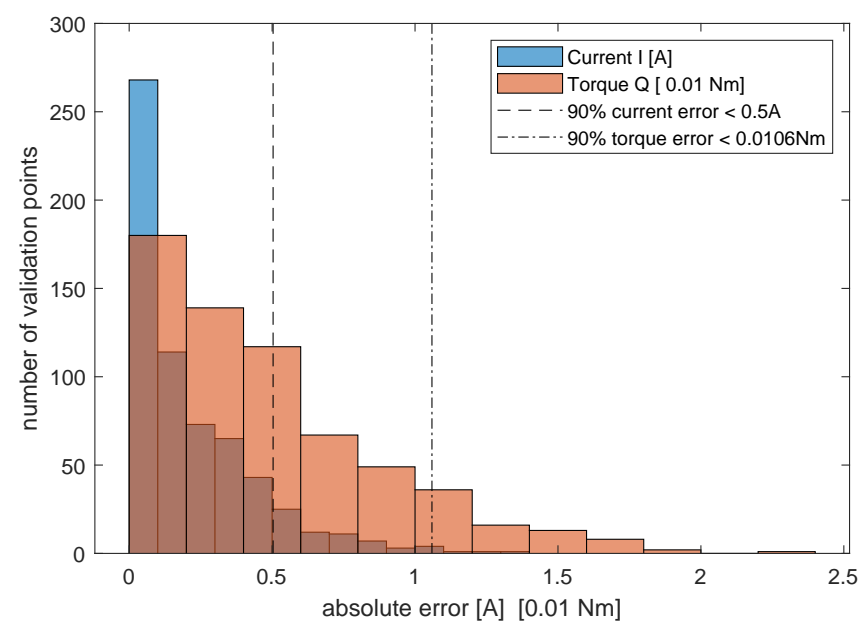

Fig. 9. Absolute current and torque error on the whole data set using the four-parameter model trained on only no-load data and the Graupner e 9x5 propeller.

\section{LOCAL IDENTIFICATION}

This section describes an approach that uses the whole data set to accurately predict torque and current of the propulsion system over its whole operating range for a given input voltage, rotational speed and throttle setting using a $3 \mathrm{D}$ lookup table.

\section{A. Procedure}

Directly interpolating between the measured points as presented in Fig. 4 would suffer from outliers or badly distributed data points. Therefore, the DC motor model described by (5-7) is used to locally identify motor constant, internal resistance and friction on a 3D grid. Locally identifying these three parameters was done by [20], but only as a function of the input voltage. To better populate a 3D grid, the data set is first remapped into a space that decouples the input variables of a multirotor propulsion system. The input voltage ranges from a fully charged battery voltage under light load to the almost depleted battery under high loads. The throttle setting can vary anywhere from zero to full throttle but the rotational speed is directly related to the former two. Without torque, the rotational speed linearly increases with voltage and the throttle setting, of which the latter was shown to linearly control the duty cycle. Therefore, mapping the data points with the rotational speed divided by the throttle setting and input voltage results in a better distribution as presented in Fig. 10.

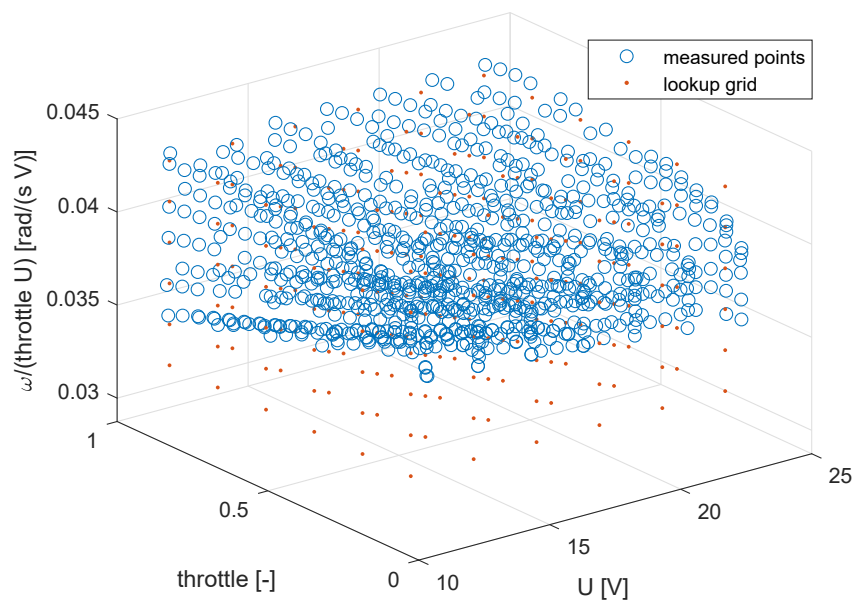

Fig. 10. Remapped input variables of the data set better populate the 3D lookup table.

Next a 3D lookup grid of query points is created and $K$, $R$ and $Q_{f}$ are fitted through the nearest neighbouring data points. For each of the query points, the distance between this query point and its neighboring data points is calculated. This is done defining a non-dimensional space around the query point by dividing the input voltage of the surrounding data points by the input voltage for this query point and dividing the rotational speed of the surrounding data points by the rotational speed of this query point. To demonstrate the validity of this method over the whole operating range of the propulsion system, $20 \%$ of the data points are randomly removed from the set used for fitting. During the fitting, less weight was given according to the distance. Fig. 11 presents the resulting locally identified parameters for each query point in the lookup grid. The manufacturer specified a motor constant of $900 \mathrm{KV}$ but in practice this parameter can vary considerably from this value [14]. The estimated motor constant shows only little variation which is in agreement 
with [17]. With values between 800 and $850 K V$, this is consistently lower than the manufacturer's specification. This is in agreement with [23] where this difference is explained by losses compared to the theoretical motor design.

The estimated interal resistance varies between roughly $0.22 \Omega$ to $0.36 \Omega$ and the estimated constant friction torque between $0 \mathrm{Nm}$ and $0.01 \mathrm{Nm}$. Notice that the identified internal resistance increases with the input voltage which agrees with (10) from the proposed model.
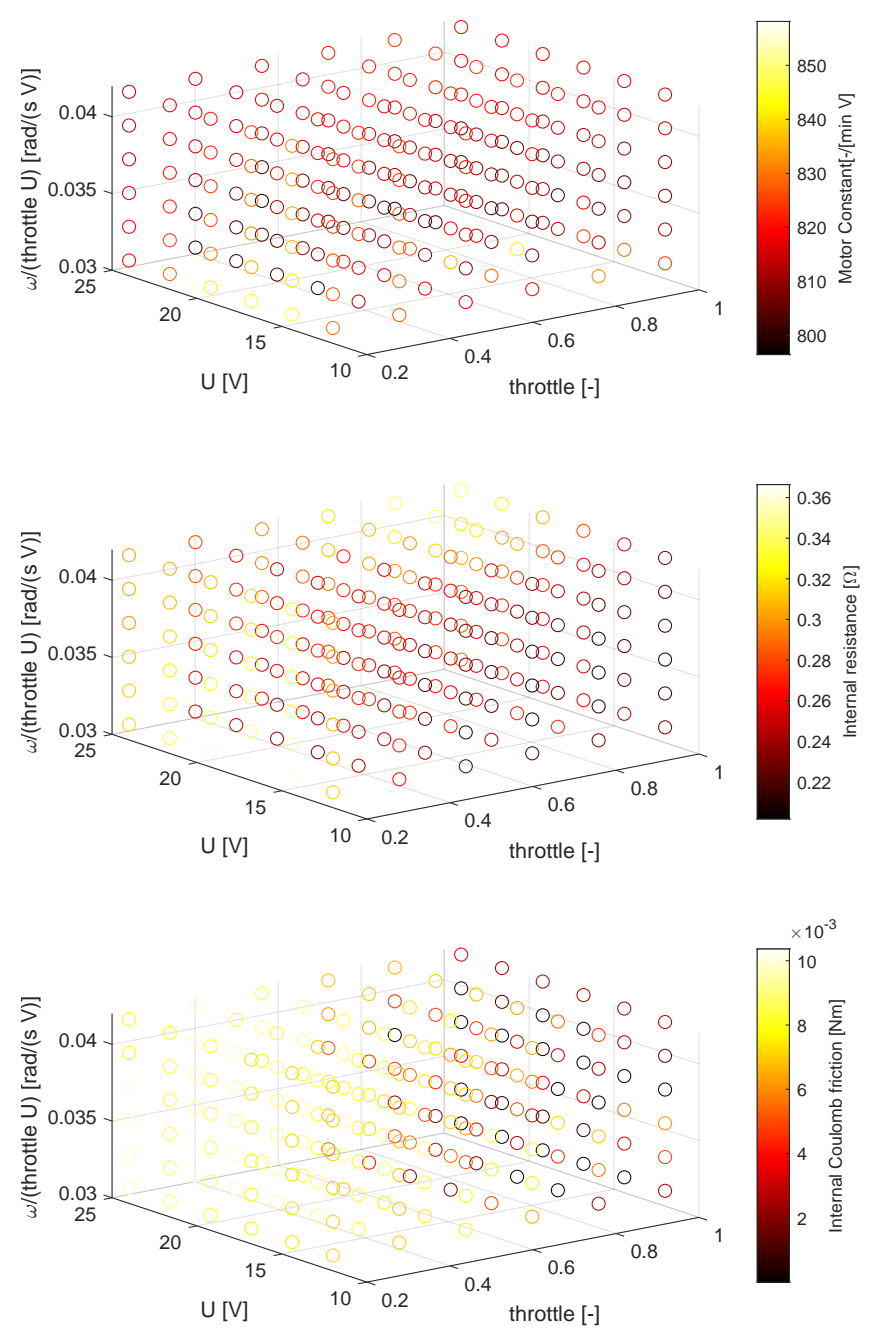

Fig. 11. Locally identified parameters for each point in the 3D grid.

\section{B. Validation}

Using the three lookup tables from Fig. 11, trilinear interpolation results in the three DC motor model parameters from which current and torque is calculated for each of the withheld validation point from the data set. Fig. 12 presents the distribution of the absolute error on both estimated current and torque for these validation points. For $90 \%$ of the validation points, the error on the current is less than $0.15 \mathrm{~A}$ and on the torque less than $0.005 \mathrm{Nm}$ which is very small compared to the variations we can expect between different experiments as was shown in Fig. 3.

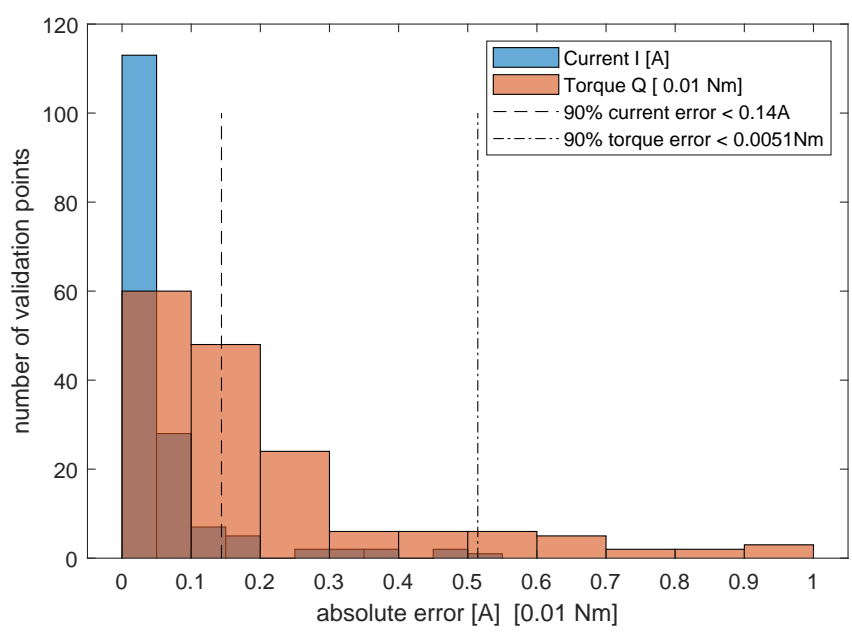

Fig. 12. Absolute current and torque error of the locally fitted threeparameter DC motor model for $20 \%$ validation points randomly distributed over the whole data set.

\section{CONCLUSIONS \& FUTURE WORK}

This paper presented two methods for calculating torque and current of a multirotor motor with ESC using the input voltage, rotational speed and throttle signal. To this end, a high-resolution data set was created spanning the feasible operating range of a motor with ESC combination. A proposed four-parameter model that was identified based on data of only one propeller and data without propeller succeeded in accurately calculating both torque and current for the whole operating range. The local identification approach based on three parameters results in a 3D lookup table suited for a highly accurate and fast prediction of current and torque. Table II summarizes the achieved accuracies of the two methods

TABLE II

OVERVIEW ACHIEVED ACCURACIES

\begin{tabular}{|c|c|c|c|}
\hline & $\begin{array}{c}\text { general fit with } \\
\text { 1 propeller }\end{array}$ & $\begin{array}{c}\text { general fit with } \\
\text { 6 propellers }\end{array}$ & $\begin{array}{c}\text { local fit with } \\
\text { 7 propellers }\end{array}$ \\
\hline $90 \% \Delta I$ & $<0.5 \mathrm{~A}$ & $<0.44 \mathrm{~A}$ & $<0.14 \mathrm{~A}$ \\
\hline $90 \% \Delta Q$ & $<0.0106 \mathrm{Nm}$ & $<0.0095 \mathrm{Nm}$ & $<0.0051 \mathrm{Nm}$ \\
\hline
\end{tabular}

In future work, several other motor and ESC combinations will be tested. The data can be used to further validate or improve the proposed four-parameter model for a general fit. A three-phase power analyser can be used to measure current and voltage at motor terminals to improve the models. Temperature effects could be included to make the model suited for different environmental and cooling conditions. The high-resolution data set will be further used to create highly accurate lookup tables for torque prediction of the motor and ESC combinations used in experiments designed for aerodynamic identification of multirotor rotors and body. The accurate virtual torque sensing will facilitate the identification of propellers in oblique flow during flight and allow better state estimation during flight. 


\section{REFERENCES}

[1] Sartori, Daniele, and Wenxian Yu. "Experimental Characterization of a Propulsion System for Multi-rotor UAVs." Journal of Intelligent \& Robotic Systems 96, no. 3-4 (2019): 529-540.

[2] Khan, Waqas, and Meyer Nahon. "A propeller model for general forward flight conditions." International Journal of Intelligent Unmanned Systems (2015).

[3] Gill, Rajan, and Raffaello DAndrea. "Computationally Efficient Force and Moment Models for Propellers in UAV Forward Flight Applications." Drones 3, no. 4 (2019): 77.

[4] Theys, Bart, Grigorios Dimitriadis, Patrick Hendrick, and Joris De Schutter. "Experimental and numerical study of micro-aerial-vehicle propeller performance in oblique flow." Journal of Aircraft 54, no. 3 (2017): 1076-1084.

[5] Brandt, J.B., Deters, R.W., Ananda, G.K. and Selig, M.S. (2005), UIUC propeller data site, available at: http://mselig.ae.illinois.edu/props/propDB.html (accessed August 10, 2015).

[6] Theys, Bart, Gregorius Dimitriadis, Thomas Andrianne, Patrick Hendrick, and Joris De Schutter. "Wind tunnel testing of a VTOL MAV propeller in tilted operating mode." In 2014 International conference on unmanned aircraft systems (ICUAS), pp. 1064-1072. IEEE, 2014.

[7] Kolaei, Amir, Devin Barcelos, and Gtz Bramesfeld. "Experimental analysis of a small-scale rotor at various inflow angles." International Journal of Aerospace Engineering 2018 (2018).

[8] Davis, Edwin, and Paul EI Pounds. "Direct sensing of thrust and velocity for a quadrotor rotor array." IEEE Robotics and Automation Letters 2, no. 3 (2017): 1360-1366.

[9] Gill, Rajan, and Raffaello D'Andrea. "Propeller thrust and drag in forward flight." In 2017 IEEE Conference on Control Technology and Applications (CCTA), pp. 73-79. IEEE, 2017.

[10] Six, Damien, Sbastien Briot, Julian Erskine, and Abdelhamid Chriette. "Identification of the Propeller Coefficients and Dynamic Parameters of a Hovering Quadrotor From Flight Data." IEEE Robotics and Automation Letters 5, no. 2 (2020): 1063-1070.
[11] Schiano, Fabrizio, Javier Alonso-Mora, Konrad Rudin, Paul Beardsley, Roland Y. Siegwart, and Bruno Sicilianok. "Towards estimation and correction of wind effects on a quadrotor UAV." In IMAV 2014: International Micro Air Vehicle Conference and Competition 2014, pp. 134-141. International Micro Air Vehicle Conference and Competition 2014 (IMAV 2014), 2014.

[12] Theys, Bart, and Joris De Schutter. "Forward flight tests of a quadcopter unmanned aerial vehicle with various spherical body diameters." International Journal of Micro Air Vehicles 12 (2020): 1756829320923565.

[13] Prudden, S., A. Fisher, M. Marino, and S. Watkins. "An investigation into the effects of rotor wake interference on quadrotor UAS forward flight performance." In AIAC18: 18th Australian International Aerospace Congress (2019): HUMS-11th Defence Science and Technology (DST) International Conference on Health and Usage Monitoring (HUMS 2019): ISSFD-27th International Symposium on Space Flight Dynamics (ISSFD), p. 344. Engineers Australia, Royal Aeronautical Society., 2019.

[14] Corke, P. "In situ measurement of robot motor electrical constants." Robotica 23, no. 14 (1996): 433-436.

[15] Swevers, Jan, Walter Verdonck, Birgit Naumer, Stefan Pieters, and Erika Biber. "An experimental robot load identification method for industrial application." The International Journal of Robotics Research 21, no. 8 (2002): 701-712.

[16] Gong, Andrew, and Dries Verstraete. "Experimental testing of electronic speed controllers for UAVs." In 53rd AIAA/SAE/ASEE Joint Propulsion Conference, p. 4955. 2017.

[17] Gong, Andrew, Rens MacNeill, and Dries Verstraete. "Performance testing and modeling of a brushless dc motor, electronic speed controller and propeller for a small uav application." In 2018 Joint Propulsion Conference, p. 4584. 2018.

[18] Gabriel, Darren Lance, Johan Meyer, and Francois Du Plessis. "Brushless DC motor characterisation and selection for a fixed wing UAV." In IEEE Africon'11, pp. 1-6. IEEE, 2011.

[19] McDonald, Robert. "Electric motor modeling for conceptual aircraft design." In 51st AIAA Aerospace Sciences Meeting including the New Horizons Forum and Aerospace Exposition, p. 941. 2013. 
[20] Lundström, David, Kristian Amadori, and Petter Krus. "Validation of models for small scale electric propulsion systems." In 48th AIAA Aerospace Sciences Meeting Including the New Horizons Forum and Aerospace Exposition, p. 483. 2010.

[21] "Series 1580 Dynamometer Datasheet", accessed October 14 2020, https://cdn-docs.rcbenchmark.com/datasheets/series-1580/DatasheetRCbenchmark-Series-1580.pdf.

[22] Chan, Hoi Lam, and Kam Tim Woo. "Design and control of small quadcopter system with motor closed loop speed control." International Journal of Mechanical Engineering and Robotics Research 4, no. 4 (2015): 287-292.

[23] Khan, Waqas, and Meyer Nahon. "Toward an accurate physics-based UAV thruster model." IEEE/ASME Transactions on Mechatronics 18, no. 4 (2013): 1269-1279.

[24] Lennart, Ljung. "System identification: theory for the user." PTR Prentice Hall, Upper Saddle River, NJ (1999): 1-14. 